APJL, ACCEPTED (30 MAY 2007)

Preprint typeset using LTEX style emulateapj v. 08/22/09

\title{
SUPERLUMINAL RADIO FEATURES IN THE M87 JET AND THE SITE OF FLARING TEV GAMMA-RAY EMISSION
}

\author{
C. C. Cheung ${ }^{1,2}$, D. E. HARris ${ }^{3}$, Ł. STAWARZ $^{2,4}$ \\ ApJL, accepted (30 May 2007)
}

\begin{abstract}
Superluminal motion is a common feature of radio jets in powerful $\gamma$-ray emitting active galactic nuclei. Conventionally, the variable emission is assumed to originate near the central supermassive black-hole where the jet is launched on parsec scales or smaller. Here, we report the discovery of superluminal radio features within a distinct flaring X-ray emitting region in the jet of the nearby radio galaxy M87 with the Very Long Baseline Array. This shows that these two phenomenological hallmarks - superluminal motion and highenergy variability - are associated, and we place this activity much further $(\geq 120 \mathrm{pc})$ from the "central engine" in M87 than previously thought in relativistic jet sources. We argue that the recent excess very high-energy TeV emission from M87 reported by the H.E.S.S. experiment originates from this variable superluminal structure, thus providing crucial insight into the production region of $\gamma$-ray emission in more distant blazars.

Subject headings: Galaxies: active — galaxies: jets — galaxies: individual (M87) — radio continuum: galaxies — radiation mechanisms: nonthermal
\end{abstract}

\section{INTRODUCTION}

The proximity of M87 ( $D=16 \mathrm{Mpc}$; Tonry 1991) makes it one of the best systems to study relativistic jets at high linear resolution (Fig. 11). Our observations with the Chandra Xray Observatory isolated short (month) timescale variability (Harris et al. 2006) in a jet region previously dubbed 'HST-1', which is separated by $0.86^{\prime \prime}$ ( $60 \mathrm{pc}$, projected) from the central "active" supermassive black hole (SMBH). The variability culminated in a factor $>50 \mathrm{X}$-ray outburst making HST-1 the brightest X-ray source in the galaxy for a few years. Figure 2 shows a lightcurve from 2000 to the end of 2006.

Observations with the Hubble Space Telescope (HST) and the Very Large Array (VLA) show comparable activity in the optical and at radio frequencies (Perlman et al. 2003; Harris et al. 2006). After the detection of appreciable radio flux in HST-1 from our first season of VLA observations (in 2003), we began to monitor the jet at higher (sub-parsec) resolution with the $\mathrm{NRAO}^{5}$ Very Long Baseline Array (VLBA) at three frequencies $(0.33,0.61$, and $1.7 \mathrm{GHz})$ commencing in Jan. 2005. This was the period at which the X-ray and optical intensities were peaking, and the radio intensity plateaued (Fig. 2). Here, we report on the highest resolution $(1.7 \mathrm{GHz})$ observations which resolve dynamic structures within the flaring X-ray region, including the discovery of superluminal motion in multiple knots. The implications for observations of more distant $\gamma$-ray emitting relativistic jets are discussed.

\section{DESCRIPTION OF OBSERVATIONS}

Each of our nine $V L B A$ runs consisted of six one-hour integrations of M87 over an $8 \mathrm{hr}$ period, with calibrator observations interleaved. Four adjacent $8 \mathrm{MHz}$ bandwidth channels

\footnotetext{
${ }^{1}$ Jansky Postdoctoral Fellow of the National Radio Astronomy Observatory; teddy3c@stanford.edu.

${ }^{2}$ Kavli Institute for Particle Astrophysics and Cosmology, Stanford University, Stanford, CA 94305; stawarz@ slac.stanford.edu.

${ }^{3}$ Harvard-Smithsonian Center for Astrophysics, 60 Garden St., Cambridge, MA 02138; harris@cfa.harvard.edu.

${ }^{4}$ Astronomical Observatory, Jagiellonian University, ul. Orla 171, 30-244 Kraków, Poland.

${ }^{5}$ The National Radio Astronomy Observatory is operated by Associated Universities, Inc. under a cooperative agreement with the National Science Foundation.
}

were used, centered at $1.667 \mathrm{GHz}$. The full 10 antenna array was used except in Jan. 2005 (missing Hancock antenna), May 2005 (missing Saint Croix), and May 2006 (missing Pie Town). The data were calibrated using NRAO's $V L B A$ data calibration pipeline in AIPS and post-processed with a combination of DIFMAP and AIPS. The rms noise in the $V L B A$ images are within $15 \%$ of the theoretically predicted value for the full VLBA $(0.053 \mathrm{mJy} / \mathrm{bm})$. The images have been restored with a common beam (Fig. 3) which is within $10 \%$ of the uniformly weighted beams of all but the May 2005 epoch.

Over the inner jet, the new sequence of $V L B A$ images shows previously known radio structure out to $\sim 250$ mas from the core (Biretta \& Junor 1995; Dodson et al. 2006) with additional transversely resolved emission between $\sim 200-450$ mas (Fig. 11). The central position angle $\left(\mathrm{PA} \sim 290^{\circ}\right)$ of the first $\sim 100$ mas portion of the jet aligns roughly with the largescale VLA jet. Knot 'HST-1' is further downstream and is offset from the central jet being aligned with the projection of the northern edge of the resolved $\sim 200-450$ mas jet (i.e., $\mathrm{PA} \approx 294.5^{\circ}$ ).

A short $2.3 \mathrm{GHz}$ VLBA observation obtained in Jul. 2004 by the USNO ${ }^{6}$ (Fey \& Charlot 2000) detected only the two brightest features in HST-1 seen in our early VLBA images allowing us to trace these structures back by $\sim 1 / 2$ year before our program commenced. Further archival VLBI observations of varying quality as far back as in 2000 (e.g., Dodson et al. 2006) did not give any significant detections of HST-1. We registered the images from the different epochs on the position of the maximum of the radio core (Fig. 1). Based on the stability of the structure of the inner jet (Dodson et al. 2006) over the 10 total epochs of observations, the core position is determined to be aligned to a fraction of the common beam over the two year period.

With the images registered, the upstream (eastern) edge which we call 'HST-1d', is the dominant feature in the HST1 complex at the early epochs. The later epoch images reveal the emergence of a radio knot (HST-1c) moving downstream from HST-1d at a rate of $4.48 \pm 0.42 \mathrm{mas} / \mathrm{yr}\left(\beta_{\mathrm{app}}=\right.$ $1.14 \pm 0.14 c$ ) at a PA of $279^{\circ}$ and the peak radio surface

\footnotetext{
${ }^{6}$ This research has made use of the United States Naval Observatory Radio Reference Frame Image Database (RRFID)
} 


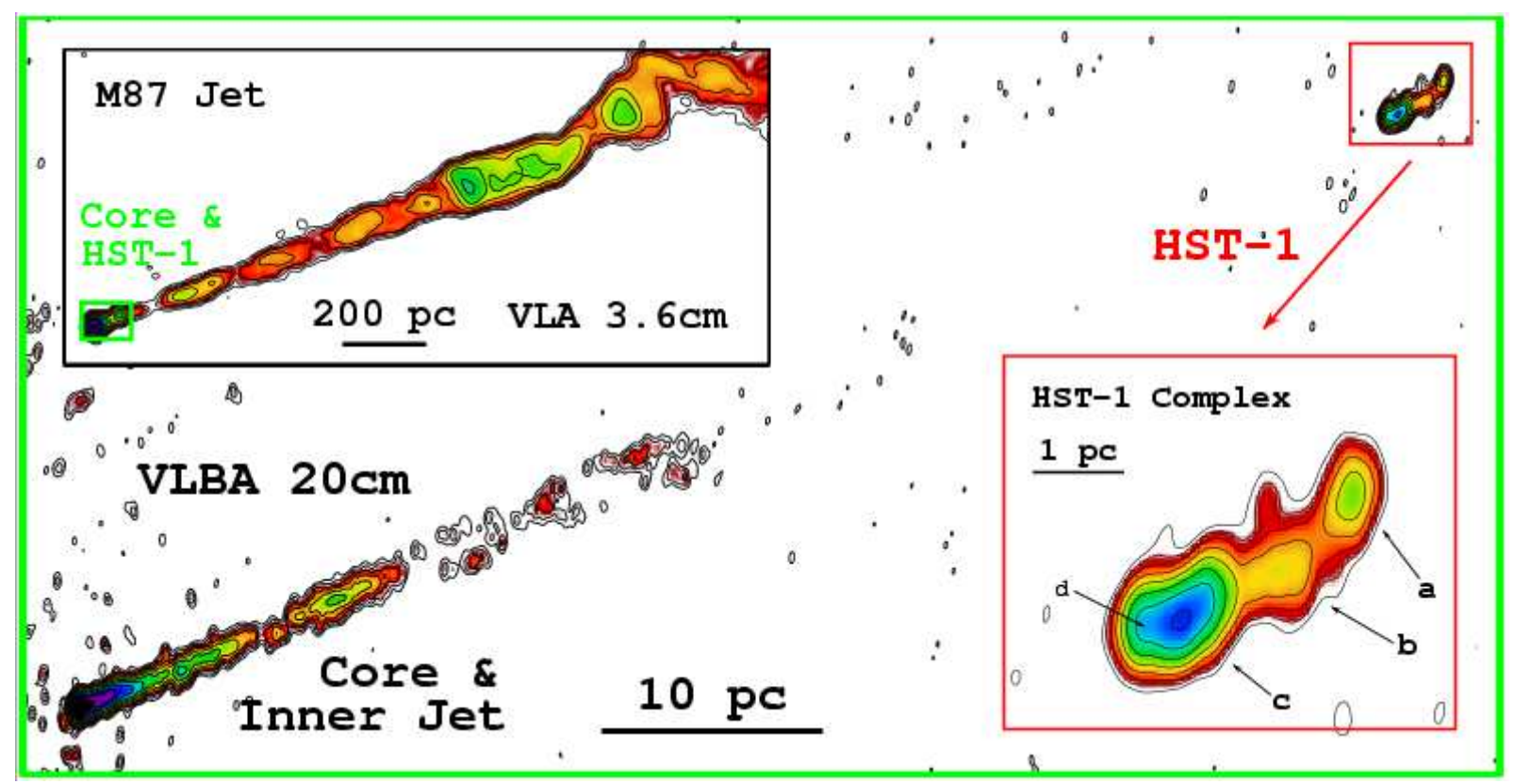

FIG. 1.- Multi-scale images of the M87 jet. Our VLBA images cover the inner $\sim 1^{\prime \prime}$ of the jet, as indicated by the corresponding green box in our VLA $3.6 \mathrm{~cm}$ image (from Dec. 2004; upper left inset) of the large-scale jet. The bottom right inset is a zoom-in of the HST-1 complex as imaged with the VLBA $(1 \mathrm{pc}=13$ mas); the lowest contour is $0.3 \mathrm{mJy} / \mathrm{bm}$.

brightness decays by only $\sim 20 \%$ over a 1 year period. Sometime between Dec. 2005 and Feb. 2006, knot HST1c evidently splits into two roughly equally bright features: a faster moving component $(\mathrm{c} 1 ; 4.3 \pm 0.7 c)$ and a slower moving trailing feature $(\mathrm{c} 2 ; 0.47 \pm 0.39 c)$. Between the two years, the location of HST-1d is basically stationary to within $\sim 2$ mas (i.e., its motion is $<0.25 c$ ) at 860 mas from the core and is the apparent point of origin of the superluminal ejections.

The most distant knot (HST-1a) is well-isolated from the other structures in every observation. We observe it moving downstream at $2.49 \pm 0.25 \mathrm{c}$ at $\mathrm{PA}=295^{\circ} \pm 8^{\circ}$ (basically radial) until Dec. 2005 when it appears to decelerate to $1.41 \pm 0.49 c$ at a smaller PA of $289^{\circ}$ (although the PA change is apparent in Figure 4, it is not statistically significant). A fainter feature (HST-1b), identifiable beginning 2005, trails HST-1a at an identical speed $(2.52 \pm 0.14 c)$ with a non-radial trajectory (a smaller PA of $279^{\circ} \pm 6^{\circ}$ ). Feature c1 (see above) actually ends up in Jul. 2006 where HST-1b was first detected in Jan. 2005.

Previous reports of motions up to $\sim 6 \mathrm{c}$ from HST-1 were based on yearly HST optical monitoring (Biretta et al. 1999) from 1994 to 1998 but pertained to emission further downstream from the VLBA structures discussed here. Furthermore, our motions are apparent on month timescales rather than years because of the higher spatial resolution of our $V L B A$ data. The extent of the radio emission in HST-1 is only $\sim 40$ mas, roughly 3 pixels across in the Biretta et al. (1999) HST FOC images; a Chandra ACIS pixel is $\sim 30 \times$ larger.

\section{DISCUSSION AND SUMMARY}

The importance of the superluminal motions reported here is twofold. First and foremost, the HST-1 complex is wellisolated from the nucleus and the rest of the jet, so the outbursting higher-energy (X-ray, optical) emission isolated by Chandra and HST can be uniquely attributed to the region resolved by our VLBA observations (Fig. 1). While VLBI detections of superluminal motions are now commonplace in more distant relativistic jet sources (e.g., Kellermann et al. 2004), the physical link to the higher-energy activity has not been possible previously because of the lack of comparably high spatial resolution (e.g., Jorstad et al. 2001). Second, contrary to conventional wisdom, this 'blazar'-like activity is clearly displaced from the central engine by $\geq 120 \mathrm{pc}$ (deprojected; see $\S 3.2$. Thus, at least in the case of M87, the observed hallmarks of blazar behavior are not directly associated with the immediate vicinity of the SMBH where the jet is launched (Junor et al. 1999). Without the comparably high linear resolution, an analogous sequence of events in a more distant source would be associated with the base (i.e., sub-pc to fewpc scales) of the jet where the 'non-jetted' contribution from the active galactic nucleus (AGN) to the observed X-rays (such as from the accretion disk; Marscher et al. 2002) would contaminate the lightcurves.

\subsection{The Origin of VHE $\gamma$-ray Emission in $M 87$}

At even higher-energies, the H.E.S.S. collaboration recently reported flaring $\mathrm{TeV}$ emission from M87 (Aharonian et al. 2006). This emission revealed gradual (year timescale) variability, with a maximum coinciding with the peak of the radio-to-X-ray activity detailed in HST-1 (mid-2005; Fig. 2) suggesting a link between these flares. However, the HST1 knot was dismissed as a possible production site of the $\mathrm{TeV}$ emission (Aharonian et al. 2006) because of the short (days) timescale $\mathrm{TeV}$ variability detected on top of the longer timescale variability. Such rapid variations were considered unlikely for HST-1 since they imply (through the causality argument) an emission region $\operatorname{size}^{7} R_{\text {var }} \lesssim 0.002 \delta$ pc. We can neither claim nor reject the presence of $\sim$ day time-scale variability in our optical or X-ray data for HST-1 due to insufficient sampling. However, the VLBA data show the compact knots in HST-1 to be essentially unresolved with semi-minor axes $<0.15 \mathrm{pc}$, and the current optical/X-ray variability data constrain $R_{\mathrm{var}} \lesssim 0.022 \delta \mathrm{pc}$ (see below), approaching the size

\footnotetext{
7 The Doppler factor is $\delta=1 / \Gamma\left(1-\sqrt{1-\Gamma^{-2}} \cos \theta\right)$, where $\Gamma$ is the bulk Lorentz factor, and $\theta$ is the angle to the line of sight.
} 


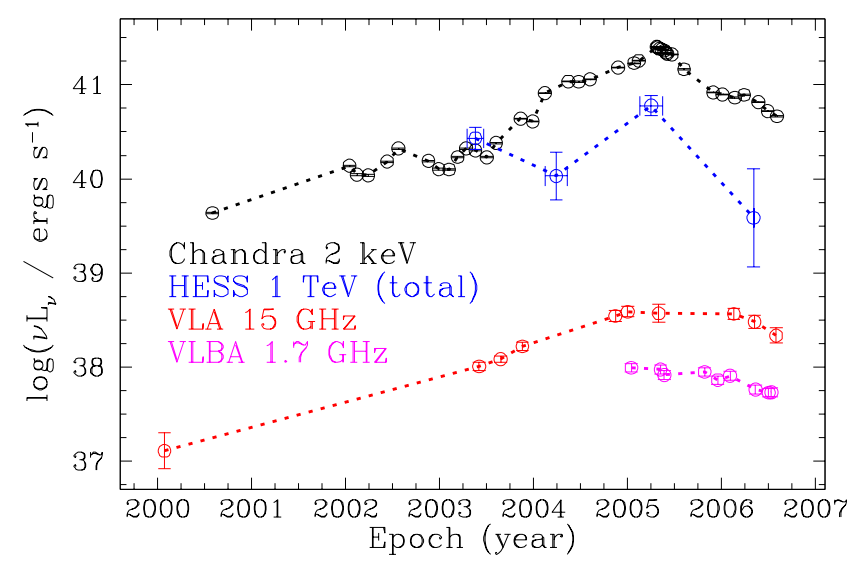

FIG. 2.- Lightcurves of the total TeV emission from M87 (taken from Aharonian et al.2006) and of the jet knot HST-1 (X-ray and two radio bands). The $2 \mathrm{keV}$ and $15 \mathrm{GHz}$ data up to $\sim$ Aug. 2005 were previously published in Harris et al. (2006). Our subsequent observations now show the X-ray intensity of HST-1 declining steeply, similar to the total TeV emission from M87. The $1.7 \mathrm{GHz} V L B A$ points are integrated from the entire HST-1 complex.

limits set by the variability of the $\mathrm{TeV}$ emission.

Here, we suggest HST-1 as a plausible site for the production of a dominant portion of the detected VHE TeV emission due to inevitable inverse-Compton upscattering off ambient photon fields (e.g., starlight) by the electrons producing the flaring synchrotron optical-to-X-ray emission. This was in fact proposed by Stawarz et al. (2006) in discussing earlier (2004 and before) TeV detections of M87. The connection is strengthened by the fact that the maxima of the total $\mathrm{TeV}$ flux density from M87 and synchrotron (HST-1 only) flares observed in 2005 were coincident, and that their luminosities are comparable (Fig. 2). In addition, the $0.4-10 \mathrm{TeV}$ emission from M87 at this time is well described by a single power-law with spectral index $\alpha_{\gamma}=1.2 \pm 0.15\left(S_{\nu} \propto \nu^{-\alpha}\right)$, while the optical-to-X-ray power-law slope of HST-1 during the same period is similar: $\alpha_{\mathrm{OX}}=0.99 \pm 0.03$. These observations are consistent with a common origin for the flaring radio through $\gamma$-ray emission from HST-1 as outlined in more detail in Stawarz et al. (2006).

The above conclusion is supported by the lack of any other plausible production site for the VHE $\gamma$-ray emission in M87. For example, the innermost (sub-pc scale) jet region is characterized by only small-amplitude optical/X-ray variability (Harris et al. 2006) and only mildly relativistic radio features (Ly et al. 2007). As argued also by Aharonian et al. (2006), this is inconsistent with models for the generation of VHE emission in the unresolved core (Georganopoulos et al. 2005). Instead, Aharonian et al. (2006) considered curvature radiation of ultra-high energy protons (Levinson 2000; Boldt \& Loewenstein 2000) accelerated by a strong magnetic field in the closest vicinity $\left(\sim 3 R_{g}\right)$ of the SMBH at the center of M87. However, this interpretation is problematic also due to the fact that the nearest environments of active SMBHs are expected to be opaque to $\mathrm{TeV}$ photons due to photon-photon $(\gamma \gamma)$ annihilation on ambient photon fields such as from the accretion disk.

In M87, the $\gamma$-ray photons in the energy range covered by H.E.S.S. $(\varepsilon=1-10 \mathrm{TeV})$ interact mostly with photons emitting at $\nu_{0} \sim 2 m_{e}^{2} c^{4} / h \varepsilon \sim 10^{13-14} \mathrm{~Hz}$, i.e., infrared to optical ones. Quantitatively, the optical depth for the $\gamma \gamma$ annihilation process can be approximated by $\tau_{\gamma \gamma} \approx$ $(1 / 3) \sigma_{\mathrm{T}} r n_{0}$, where $\sigma_{\mathrm{T}}$ is the Thomson cross-section. The number density $n_{0}$ of the $h \nu_{0}$ nuclear photons within a spher-

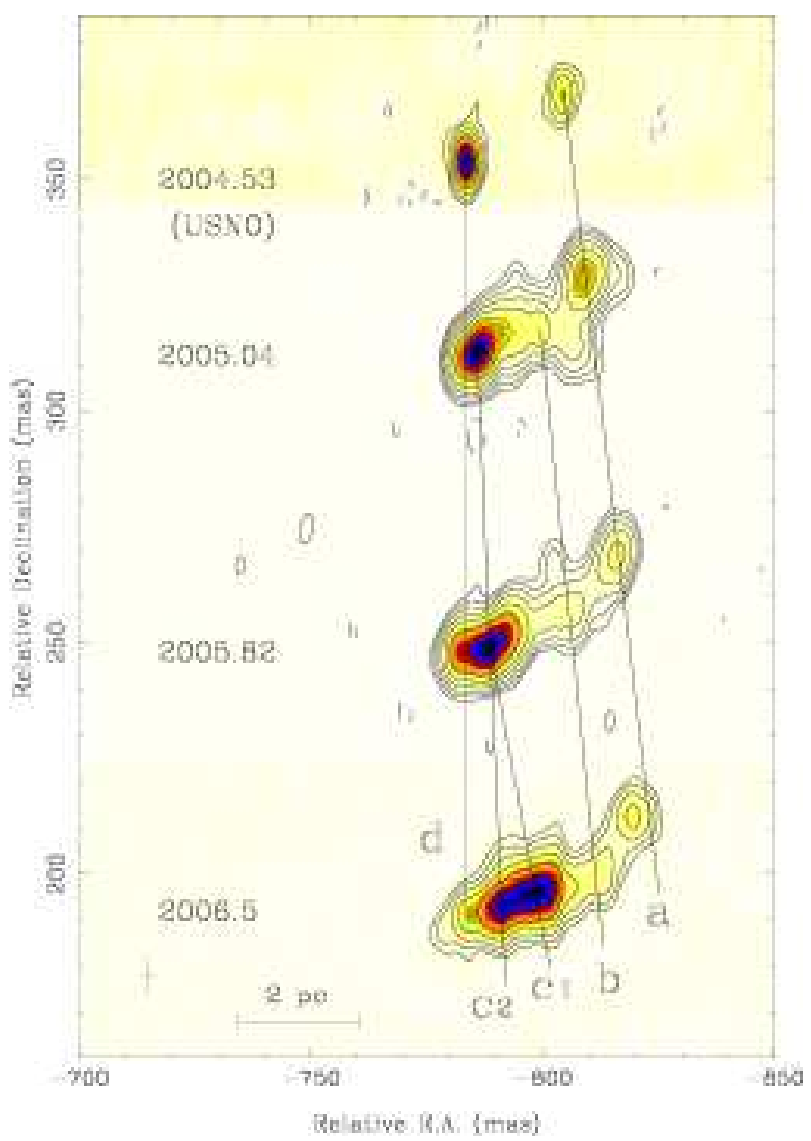

FIG. 3.- Representative sequence of $V L B A$ images of knot HST-1 (see Fig. 1) restored with a 8.0 mas $\times 3.4$ mas $(0.62 \mathrm{pc} \times 0.26 \mathrm{pc})$ beam at $\mathrm{PA}=3^{\circ}$ (bottom left). The vertical spacing is proportional to time elapsed and straight lines trace the motions of the different features (note the apparent deceleration of knot "a"). The first epoch is a shorter $2.3 \mathrm{GHz}$ dataset resulting in the excess background; subsequent ones are full track $1.7 \mathrm{GHz}$ observations.

ical volume around the SMBH of radius $r$ is related to the monochromatic luminosity $L_{0}$ by $h \nu_{0} n_{0}=L_{0} / 4 \pi r^{2} c$ (the main contributor to the photon energy density is judged to be from the accretion disk). Hence one can find, $\tau_{\gamma \gamma} \approx$ $200\left(L_{0} / 10^{40} \mathrm{erg} \mathrm{s}^{-1}\right)\left(\nu_{0} / 10^{13} \mathrm{~Hz}\right)^{-1}\left(r / R_{g}\right)^{-1}$. The considered region would be transparent to the TeV photons only if $\tau_{\gamma \gamma}<1$. However, at $r \sim(3-10) \times R_{g}$ we expect $\tau_{\gamma \gamma} \gg 1$, since the observed luminosity of the M87 nucleus (Kharb \& Shastri 2004; Whysong \& Antonucci 2004) at $\nu_{0}=$ $10^{13-14} \mathrm{~Hz}$ is $L_{0} \gtrsim 3 \times 10^{40} \mathrm{erg} \mathrm{s}^{-1}$, consistent with ADAF approximations of the accretion disk (Di Matteo et al. 2003). With the central region of the AGN excluded as a plausible site of the TeV emission, the flaring HST-1 knot is the most probable point of origin. Therefore, in addition to the flaring synchrotron (radio-to-X-ray) emission, the superluminal radio structures detailed here can also be associated with VHE $\gamma$-ray activity.

\subsection{HST-1 as a Recollimation Shock}

A natural question to ask is if the position of the HST-1 knot in the M87 jet is 'special' in some way. In other words, what determines the location and flaring behavior of this extremely compact and variable feature within the relativistic outflow? The kinematics of the superluminal radio components (in particular, the $4.3 \mathrm{c}$ motion in $\mathrm{c} 1$ ) constrain the jet at the location of HST- 1 to be aligned at $\theta \leq 26 \pm 4^{\circ}$, and re- 


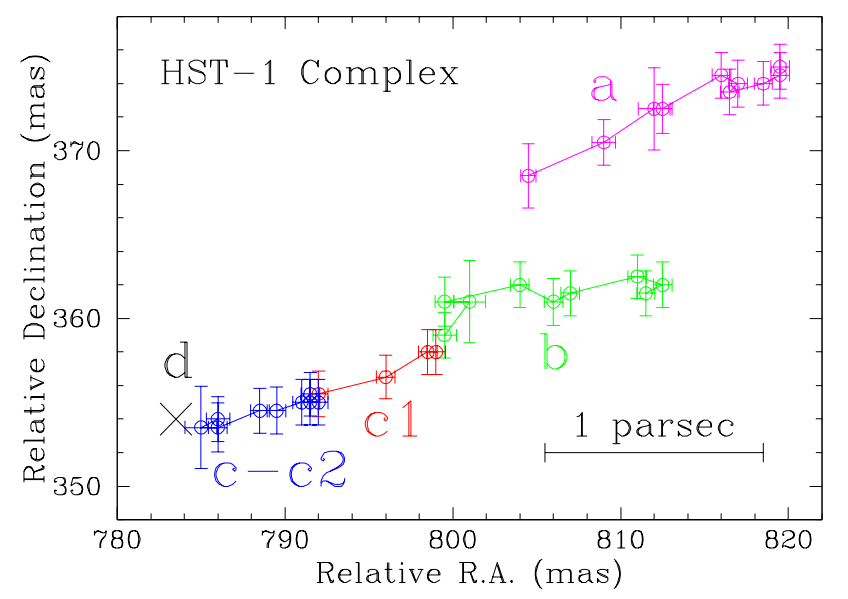

FIG. 4.- Positions of radio features in the HST-1 complex relative to the core (at origin) from our VLBA data (see Fig. 3]. The positional errors are $1 / 6$ th of the uniform weighted beam-sizes. Component ' $c$ ' emanates from the basically stationary feature 'd' (black cross) and splits into c1 and c2. All components are moving downstream (upper right).

quires $\Gamma \geq \sqrt{\beta_{\text {app }}^{2}+1}=4.4$, and $\delta \gtrsim 2$. The HST-1 region is therefore $\geq 120 \mathrm{pc}$ (deprojected assuming $\theta=30^{\circ}$ ). For a SMBH with $\mathcal{M}_{\mathrm{BH}} \approx 3 \times 10^{9} M_{\odot}$ (Macchetto et al. 1997), this corresponds to the distance of $\gtrsim 10^{6}$ Schwarzchild radii $\left(R_{g}=G \mathcal{M}_{\mathrm{BH}} / c^{2} \approx 4.4 \times 10^{14} \mathrm{~cm} \approx 1.4 \times 10^{-4} \mathrm{pc}\right)$. At such a large distance from the active center, our X-ray observations establish flux doubling timescales $t_{\mathrm{var}}$ of 0.14 yrs (Harris et al. 2006), which constrains $R_{\mathrm{var}}<c t_{\mathrm{var}} \delta \approx 0.022 \delta$ pc.

Recently, Stawarz et al. (2006) showed that the position of the HST-1 knot agrees with the expected location of a 'reconfinement nozzle' formed within the M87 jet due to a converging shock driven by the interaction of the outflow with the interstellar medium (ISM). In this ambient medium, it was postulated that the gravitational influence of the central SMBH in the inner region of the M87 host galaxy (Lauer et al. 1992) results in an increase of the gas pressure, analogous to the forming of the observed enhanced stellar cusp (Young et al. 1978). For the temperature $k T_{\text {ism }} \approx 0.8 \mathrm{keV}$ of hot gaseous ISM (with the number density $n_{\text {ism }} \approx 0.17 \mathrm{~cm}^{-3}$ ) in the inner $(<1 \mathrm{kpc})$ parts of the galaxy, the gravitational capture radius is $R_{A}=G \mathcal{M}_{\mathrm{BH}} / c_{\mathrm{s}}^{2} \approx 100 \mathrm{pc}$, where $c_{s}$ is the appropriate sound speed (Di Matteo et al. 2003). It turns out that $\sim 100 \mathrm{pc}$ is the spatial scale of the disk of ionized gas observed in M87 by the HST (Ford et al. 1994). It was the Keplerian rotation of the inner parts of this disk which enabled a precise estimate of the black hole mass in this system (Macchetto et al. 1997).

Thus, one can surmise that a gravitationally perturbed ambient medium leads naturally to the formation of a jet feature like HST-1 at $\gtrsim 100 \mathrm{pc}$ from a central SMBH. In this scenario, the 'stationary' region HST-1d defines the opening of the nozzle. The origins of the different superluminal features can be traced back to this position at $\sim 2004.5$ (HST-1c), 2003.3 (HST-1b), and 2001.9 (HST-1a). The latter two could have originated more recently as they assume the nominal velocities of $2.5 c$; the apparent curve in trajectory and deceleration of HST-1a lead us to believe it could have been moving faster previous to our first $V L B A$ imaging observations.

The formation of a 'reconfinement nozzle' within the jet is not unique for the hydrodynamical outflow considered by Stawarz et al. (2006). It was previously shown (Tsinganos \& Bogovalov 2002) that interactions between a strongly magnetized relativistic outflow with a non-relativistic collimating magneto-hydrodynamical (MHD) wind may lead as well to the formation of a converging shock within the relativistic component. An evaluation of the exact position of this shock from the jet base is extremely model-dependent. Nevertheless, for the jet parameters anticipated by Gracia et al. (2005), who successfully explained the observed gradual collimation of the M87 jet between $10^{2}-10^{6} R_{g}$ (Junor et al. 1999) based on the Tsinganos \& Bogovalov (2002) MHD model, the expected position is near the location of the HST-1 knot. We speculate that a rapid release of the magnetic energy within the formed nozzle via magnetic reconnection may play an important role in producing the observed high energy flares and ejection of the relativistic radio blobs, somewhat analogous to coronal mass ejections from the Sun (Pick et al. 2006).

In summary, we have discovered superluminal motions of radio features in the $\mathrm{M} 87$ jet at a site remote from the central SMBH. These features appear to be associated with the remarkable flare of 2005 for which the radio, optical, and X-ray flux densities peaked at levels of $>30-50$ times that of a few years earlier. We have argued that the $\mathrm{TeV}$ excess intensity of 2005 detected from M87 by H.E.S.S. was a manifestation of the same event. Thus, most of the defining characteristics of blazars have now been shown to occur at a distance $\geq 120$ pc from the SMBH instead of from the immediate environs of the central engine, as is commonly believed to be the case. As we enter the GLAST era, ensuing studies of high-energy flares from blazars should consider such a production site as we have resolved in M87.

We thank Bill Junor for his involvement in the early stages of this project, Roger Blandford for discussions on the $\gamma$-ray opacity, and Al Marscher and Ken Kellermann (the referee) for helpful comments on the manuscript. This work was supported by NASA (D. E. H.) and by MEiN through research project 1-P03D-003-29 from 2005-2008 (Ł. S.).

\section{REFERENCES}

Aharonian, F., et al. (H.E.S.S. collaboration). 2006, Science, 314, 1424 Biretta, J. A., \& Junor, W. 1995, PNAS, 92, 11364

Biretta, J. A., Sparks, W. B., \& Macchetto, F. 1999, ApJ, 520, 621

Boldt, E., \& Loewenstein, M. 2000, MNRAS, 316, L29

Di Matteo, T., et al. 2003, ApJ, 582, 133

Dodson, R., Edwards, P. G., \& Hirabayashi, H. 2006, PASP, 58, 243

Fey, A. L., \& Charlot, P., 2000, ApJS, 128, 17

Ford, H. C., et al. 1994, ApJ, 435, L27

Georganopoulos, M., Perlman, E. S., \& Kazanas, D. 2005, ApJ, 634, L33

Gracia, J., Tsinganos, K., \& Bogovalov, S. V. 2005, A\&A, 442, L7

Harris, D. E., et al. 2006, ApJ, 640, 211
Jorstad, S. G., et al. 2001, ApJ, 556, 738

Junor, W., Biretta, J. A., \& Livio, M. 1999, Nature, 401, 891

Kellermann, K. I., et al. 2004, ApJ, 609, 539

Kharb, P., \& Shastri, P. 2004, A\&A, 425, 825

Lauer, T. R., et al. 1992, AJ, 103, 703

Levinson, A. 2000, PRL, 85, 912

Ly, C., Walker, R. C., \& Junor, W. 2007, ApJ, 660, 200

Macchetto, F., et al. 1997, ApJ, 489, 579

Marscher, A. P., et al. 2002, Nature, 417, 625

Perlman, E. S., et al. 2003, ApJ, 599, L65

Pick, M., et al. 2006, Space Science Reviews, 123, 341 
Stawarz, Ł., et al. 2006, MNRAS, 370, 981

Young, P. J., et al. 1978, ApJ, 221, 721

Tonry, J. L. 1991 ApJ, 373, L1

Tsinganos, K., \& Bogovalov, S. 2002, MNRAS, 337, 553

Whysong, D., \& Antonucci, R. 2004, ApJ, 602, 116 